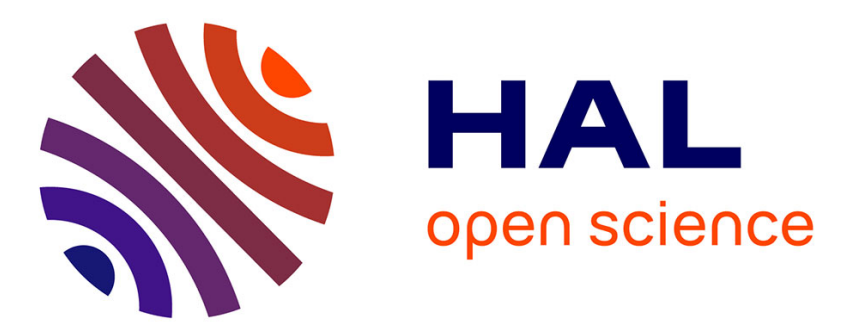

\title{
Un examen critique des liens entre le Traité des probabilités et la Théorie générale de Keynes
}

Nicolas Piluso

\section{To cite this version:}

Nicolas Piluso. Un examen critique des liens entre le Traité des probabilités et la Théorie générale de Keynes. Cahiers d'Economie Politique = Papers in political economy, 2015. hal-01399077

\section{HAL Id: hal-01399077 \\ https://hal-univ-tlse2.archives-ouvertes.fr/hal-01399077}

Submitted on 18 Nov 2016

HAL is a multi-disciplinary open access archive for the deposit and dissemination of scientific research documents, whether they are published or not. The documents may come from teaching and research institutions in France or abroad, or from public or private research centers.
L'archive ouverte pluridisciplinaire HAL, est destinée au dépôt et à la diffusion de documents scientifiques de niveau recherche, publiés ou non, émanant des établissements d'enseignement et de recherche français ou étrangers, des laboratoires publics ou privés. 


\title{
Nicolas Piluso \\ CNRS-CERTOP (UMR 5022)
}

\section{Un examen critique des liens entre le Traité des probabilités et la Théorie générale de Keynes}

Résumé : A partir des années 80, certains économistes voient dans le Traité des probabilités de Keynes un élément incontournable de son approche «fondamentaliste » fondée sur l'incertitude. D'après ces derniers, il existe des liens de complémentarité forts entre le Traité des probabilités et la Théorie générale. Pourtant, un tel projet de développement du Traité des probabilités n'a pas abouti, bien qu'on en retrouve des éléments dans le chapitre 12 de la Théorie générale. Nous tentons donc de comprendre d'une part ce qui a motivé la naissance d'un tel point de vue, et d'autre part pourquoi ce projet n'a pas été développé comme l'avaient annoncé certains économistes français. Nous montrons que l'objet d'analyse du Treatise porte sur un type d'incertitude différent de celui de la Théorie générale.

Mots clé : confiance, convention, incertitude, prévisions, probabilités.

Codes JEL: B22, B40, B50.

\begin{abstract}
From the 80s, some economists believe that the keynesian Treatise of probability is a key element of the "fundamentalist" approach based on uncertainty. According to them, there is a complementary relationship between the Treatise and the general theory. However, such a radical project based on the treatise of probability has never succeeded, although it is found in the developments of Chapter 12 of the General theory. We are trying to understand firstly what motivated the birth of such a point of view, and secondly why this project has not been developed as some French economists had announced.. We show that the object of analysis of the treatise covers a different type of uncertainty that the General theory.
\end{abstract}

Key words: Trust, convention, uncertainty, forcasts, probabilities.

JEL codes : B22, B40, B50.

\section{Introduction}

Keynes n'a pas pris conscience du rôle majeur de l'incertitude au cours de l'élaboration de la Théorie générale. Ses intuitions en la matière sont bien antérieures, puisqu'elles constituent le point de départ même de sa réflexion, alors qu'il était encore doctorant. Le Treatise on probability [1921], première grande oeuvre de Keynes, présente une théorie de la prise de décision en avenir incertain tout à fait originale. Elle semble, de prime abord, s'inscrire dans un cadre analytique extrêmement proche de celui adopté dans la Théorie générale. En effet, le vide que laisse la Théorie générale en matière de théorie des anticipations pourrait en fait être simplement expliqué par le développement d'une telle analyse dans le Treatise. D'après certains économistes (voir Postel, [2000], Ventelou [1997], Orléan [1999, 1994], Favereau [1988, 1985], Arrous [1982]), ce dernier renfermerait deux éléments indispensables au plein développement de l'approche fondamentaliste de Keynes (voir Barrere [1990], Orio\&Quiles, [1994]): d'une part, une endogénéisation des anticipations en avenir incertain, et, corrélativement, une première forme d'analyse des modes de coordination hors-prix des décisions individuelles, autrement dit, les traces d'un concept de convention. Le Treatise on probability fournit, d'après eux, les éléments d'accomplissement de l'approche fondamentaliste sur le thème majeur qui est le sien, l'incertitude.Ce point de vue est partagé également par Lawson [1985], qui estime que l'approche développée par Keynes dans le Treatise et la Théorie générale fournissent les éléments de base d'un programme de recherche : «I wish to argue that Keynes' views on uncertainty, for frombeinginnocuous or destructive of economicanalysis in general, cangiverise to a research program incorporating, 
amongstotherthings, a view of rational behaviourunderuncertaintywhichispotentiallyfruitful » (p. 909).

Pourtant, force est de constater aujourd'hui que les développements des travaux de recherche postkeynésiens font rarement appel aux concepts élaborés par Keynes dans le Treatise on probability(voir Le Heron et al., [2011]) ${ }^{1}$. Nous nous proposons donc de chercher à comprendre pourquoi un tel projet de développement des thèses initiales de Keynes est tenu en échec.

Nous analyserons tout d'abord les éléments qui créditent apparemmment, et en première analyse, le point de vue selon lequel la théorie de l'incertain manquante à laThéorie générale est présente dans le Treatise. Il s'agit de reconstituer le cheminement de Keynes dans le Treatise on probability, au terme duquel une véritable théorie de la formation des anticipations est constituée. Cette dernière permettrait d'éclairer l'ensemble des éléments de la Théorie générale ayant trait à la prévision de court et long terme; nous pourrons ainsi synthétiser la théorie keynésienne des anticipations. L'objectif de cette première partie de l'article est ainsi de rendre intelligible la position de plusieurs économistes keynésiens. Nous verrons pourquoi ces derniers identifient dans le Treatise les traces d'un concept de convention commun à celui exposé dans la Théorie générale.

Cependant, eu égard au constat mentionné plus haut, il est nécessaire de comprendre l'absence de développement significatif de ce projet de recherche. Il est lié selon nous à une erreur d'analyse fondamentale que nous mettrons en lumière dans une seconde partie.

\section{Théorie de la formation des anticipations et Théorie générale : les possibles liens de complémentarité}

\subsection{La convention dans le Treatise on probability}

La problématique sur laquelle se fonde le Treatise on probability, bien connue des commentateurs de Keynes, est d'ordre épistémologique. Keynes part du constat que dans le cours ordinaire de notre pensée, nous accordons une crédibilité certaine à des propositions dont la caractéristique est ne pas être totalement démontrable, ou encore étayée par des faits présents ou passés. Or la théorie de la connaissance et des probabilités en général ne s'intéressent qu'aux évènements, et non aux propositions; elle ne s'appuie que sur des données certaines à partir desquelles un jugement sûr sur l'avenir peut être fondé. Force est pourtant d'observer que la connaissance, dans le domaine notamment de la vie sociale, ne se fonde pas dans un tel cadre. Keynes en déduit que des pans entiers de l'activité sociale ne peuvent être que des laissés pour compte de l'épistémologie.

Ce à quoi l'auteur s'attelle dans son Treatise on probability est ainsi l'extension de la théorie de la connaissance et des probabilités aux domaines qui ne rentrent pas dans le modèle restrictif de l'approche traditionnelle. Le caractère étroit de cette dernière conception n'est pas postulé par Keynes : ce dernier nous en livre une analyse précise dont nous rappellerons les lignes directrices. Cette critique appelle un renouvellement du concept de probabilité. Ainsi Keynes développe-t-il une théorie alternative, dénommée théorie logique des probabilités, qui constitue une réponse au problème de la connaissance dans le cadre d'un

\footnotetext{
${ }^{1}$ On peut citer comme exemple d'exception à cette affirmation le travail de Roncaglia [2009].
} 
environnement incertain (que nous définirons). Cette théorie se veut générale, et à cet égard, elle ne s'oppose pas au courant traditionnel mais l'englobe.

La théorie orthodoxe n'aborde pas le problème de la formulation de propositions dont les fondements ne fournissent que des indications parcellaires et incertaines de la conclusion à laquelle elle est reliée. Et pourtant, comme Keynes le souligne, cette situation ne relève pas de l'irrationnel ou encore de l'illogisme. Il est courant d'investir ce type de raisonnement d'un "degré de croyance rationnel », dans la mesure où cette croyance est logiquement fondée. Keynes écrit à ce propos que le fondement logique d'une proposition ne tient pas à un état de connaissance parfaite des individus sur la question. Ce n'est pas parce qu'une démonstration n'est pas disponible que pour autant, la proposition dont il est question est dénuée de toute rationalité.

Keynes définit alors l'objet de son Treatise. Dans la mesure où des propositions logiques incertaines s'expriment le plus souvent en termes de probabilités, la théorie logique des probabilités va constituer la substance de la théorie générale de la logique.

Les questions qui se posent dès lors sont les suivantes : qu'est-ce que Keynes entend par relation de probabilité ? En quoi cette conception nouvelle embrasse l'approche traditionnelle et en quoi cette dernière ne peut s'appliquer au champ de l'activité sociale ?

La relation apparaît chez Keynes comme un opérateur de connaissance, dont l'objet est le lien d'implication qui unit un ensemble de prémisses d'une part, et un ensemble de conclusions d'autre part. La relation de probabilité n'exprime pas un degré de vérité ou d'erreur, mais un degré de connaissance ou, pour reprendre l'expression la plus souvent employée par Keynes dans le Treatise, un degré de croyance rationnelle. Keynes la définitcomme suit: «The premisses consist in any set of propositions $h$, and our conclusion consist of any set of proposition a, then, if a knowledge of $h$ justify a rational belief in a degree $\alpha$, we say that there is the probability-relation of degree $\alpha$ between a and $h$. This will be written $\mathrm{a} / \mathrm{h}=\alpha \gg(\mathrm{p} .11)$.

Ce que Keynes entend par « relation de probabilité » est donc un degré de connaissance et d'implication affaibli entre deux ensembles de propositions. Ainsi, lorsque la relation d'implication est totale, c'est-à-dire certaine, $a / h=\alpha=1$. Le cas de la limite inférieure signifie la contradiction entre $\mathrm{a}$ et $\mathrm{h}$, contradiction au sens où « $\mathrm{a} »$ ne peut être logiquement déduit de prémisses telles que «h». Dans tous les autres cas, lorsque $0<\alpha<1$, la relation exprime le degré d'implication adéquat de «a $»$ compte-tenu du capital informationnel « $\mathrm{h} »$.

O’Donnell [1989] relève plusieurs propriétés fondamentales de la relation de probabilité.

A la page 4 du Treatise, Keynes note que la probabilité n'est pas une propriété des propositions elles-mêmes. Ilajoute: «it is significant to call a proposition probable unless we specify the knowledge to which we are relating it» [1921, p.11]. La probabilité, autrement dit, est une relation. Elle n'a d'existence que par rapport à un corps initial de propositions et ne s'attache pas à un événement isolé. Il s'agit là d'un sillage dans lequel l'interprétation conventionnaliste a pénétré [Orléan, 1994]. La convention keynésienne serait, d'après cette dernière, le fameux point de référence indispensable à la formulation des probabilités et à l'harmonisation des anticipations. 
La relation de probabilité n'est pas un degré quelconque de croyance. Keynes insiste sur le fait qu'il est rationnel. Cela signifie qu'il est toujours logiquement fondé. A cet égard, Keynes se distingue totalement du courant subjectiviste né de la critique ramseyienne de son Treatise. Quand bien même notre auteur reconnaît l'intervention d'éléments subjectifs dans le processus décisionnel, donc dans la production de la relation de probabilité, il affirme avec vigueur l'objectivité de la relation de probabilité.

La chose ne signifie cependant pas que tous les individus, face à une situation qui leur est commune, prennent tous la même décision. Les agents n'ont pas en effet les mêmes capacités mentales d'induction et le même stock d'informations. C'est à ce point du raisonnement qu'intervient la dose de «subjectivisme » dans la pensée de Keynes. Il reste que si la probabilité $\mathrm{a} / \mathrm{h}$ est unique, la relation $\mathrm{a} / \mathrm{h}$ ' avec $\mathrm{h}$ ' identique dans le contenu et la signification mais moins fournie en termes quantitatifs, est aussi unique et objective.

Reprenons à présent les points de vue de Favereau [1985] et d'Orléan [1986]. Ré-explorant le concept d'incertitude, le dernier auteur note que l'exposé qu'en fait Keynes dans l'article de 1937 est flou. Pour Orléan, cette notion ne peut être établie qu'en rapport avec un capital cognitif existant. L'auteur ne fait ici en réalité que reprendre à son compte l'idée de Keynes selon laquelle la probabilité n'a de sens qu'eu égard à un corps de connaissances qu'elle relie à un corps de conclusions.

La convention, dans la problématique conventionnaliste, s'inscrit dans la recherche de procédures «hors-marché » de coordination. Le terme de coordination revêt ici un sens particulier : il est entendu comme le maintien, dans le cadre d'un futur dit incertain, d'une hétérogénéité minimale des anticipations, endiguant les processus de hausse ou de baisse cumulative des prix et/ou de l'activité. A ce titre, l'école des conventions semble rejoindre Keynes en affirmant :

- le caractère relatif de l'incertitude,

- l'assise sur laquelle se fonde la relation de probabilité,

- l'existence d'une «méthode conventionnelle de calcul», pour reprendre les termes de Keynes, guidant le marché.

Favereau [1985] souligne le lien étroit entre la convention keynésienne et les prémisses du Treatise On Probability. Pour le voir, il suffit de reprendre la définition formelle de la régularité lewisienne, que les conventionnalistes reprennent à leur compte :

\footnotetext{
« A regularity $\mathrm{R}$, in action and belief (souligné par nous), is convention in a population if and only if, within $\mathrm{P}$, the following six conditions hold :

- everyone conforms to $\mathrm{R}$

- everyone believes that others conform to $\mathrm{R}$

- this belief that the the other conform to R gives everyone a good and decisive reason to conform to $\mathrm{R}$ himself

- there is a general preference to general conformity

- $\mathrm{R}$ is not the only possible regularity making the last two conclusions

- finally, the various facts listed are matters of commun knowledge» [Lewis, cité par Favereau, 1985].
}

Transposée à la terminologie du Treatise, la régularité ne peut désigner que les prémisses de la relation de probabilité. Les prémisses constituent un corps de connaissances, tandis que la convention est connaissance commune. Nous pouvons donc établir que la convention n'est jamais qu'un ensemble de prémisses de la relation de probabilité, communément adopté par les agents économiques. 


\subsection{La théorie keynésienne de la formation des anticipations}

Certains commentateurs de Keynes considèrent que les anticipations, bien que jouant un rôle important dans l'analyse de la Théorie générale, ne sont pas l'objet d'une théorie constitutive. Pourtant, il est peut-être possible de trouver dans la Théorie générale des traces explicites d'une théorie des anticipations forgée par ailleurs dans le Treatise on probability. L'objet de cette section est donc d'exposer la théorie keynésienne des anticipations, en synthétisant les éléments des deux ouvrages en question. Keynes distingue en effet trois phases dans le processus de décision, qui font l'objet des trois sous-sections ci-dessous.

Première étape : la récolte des informations.

Keynes établit une double typologie de la connaissance. La première permet de définir les termes de la relation de probabilité (h et a); la seconde rend compte du degré d'incertitude, qui peut tenir tant du degré d'habileté de l'individu décideur que des informations disponibles. La seconde ayant déjà été traitée au travers de l'analyse de la signification de la relation de probabilité, nous ne nous intéresserons ici qu'à la première.

Il distingue tout d'abord la connaissance directe de la connaissance indirecte. La première a pour objet les prémisses « $\mathrm{h} »$ que l'individu s'approprie via ses sensations, ses perceptions, sa compréhension, à la lumière des enseignements de son expérience.

La connaissance indirecte au contraire est générée par la perception de relations logiques entre propositions. Elle est rendue possible par le raisonnement. Les conclusions «a » sont obtenues au travers du lien qui l'unit avec les prémisses « $\mathrm{h} »$. Ce lien est la relation de probabilité.

Seconde étape: La production de la relation de probabilité.

La première étape de la décision consiste dans la récolte des informations pertinentes pour l'anticipation. C'est là qu'intervient la prise de connaissance directe avec les prémisses informationnelles $« \mathrm{~h} »$. La seconde étape va procéder du raisonnement et de la déduction d'un lien logique d'implication entre le corps de conclusions que l'on cherche à anticiper et les prémisses initialement acquises. Toutefois, la production d'un tel lien n'est pas obtenue par une pure et simple déduction des données empiriques récoltées. Ces dernières sont considérées par Keynes comme une condition nécessaire mais non suffisante à la naissance d'une connaissance indirecte.

L'assise empirique de la connaissance est un élément essentiel de l'épistémologie de Keynes, mais pas la seule. L'intuition vient la seconder. L'auteur considère en effet que les prémisses sont composites, non seulement au sens qui a déjà été mentionné (propositions certaines/incertaines), mais aussi en ce qu'elles contiennent des données empiriques et des propositions de vérité générale. Ces derniers éléments dépassent l'expérience contingente aux individus et relèvent de l'intuition. Il est des relations logiques qui apparaissent évidentes d'elles-mêmes, et qui n'appellent pas d'autre justification que celles des vues de l'esprit. Les connaissances directes, comme ensemble de connaissances empiriques et intuitives, constituent l'ancrage du système de prise de décision, le rempart d'une régression de 
justification en justification. Sans elle, l'individu serait conduit à remonter infiniment la chaîne des causes et des effets.

Est-ce que cette intuition, qui lève l'indétermination de la décision, peut être assimilée à la convention qui est le rempart à la spécularité des anticipations ? Si convention et intuitions semblent jouer un rôle similaire, leur nature demeure néanmoins fondamentalement différente et s'exclue l'une de l'autre. La première renvoie à un modèle de l'économie alors que la seconde transcende les constructions du raisonnement. Plus essentiellement, la convention relève du choix collectif d'une représentation de l'économie tandis que l'intuition est hors de tout espace de choix, aussi bien à un niveau individuel qu'à un niveau collectif (si tant est qu'on puisse parler d'intuition collective).

Troisième étape: la pondération de la relation de probabilité par l'état de confiance.

Keynes introduit dans le chapitre 6 du Treatise un concept qui prendra au fil des années une importance de plus en plus grande dans son analyse économique des décisions. Hésitant quant à l'importance à lui donner dans le Treatise, Keynes en fait un élément essentiel des anticipations dans la Théorie générale. Il s'agit du concept de "poids des arguments » ou « poids des jugements », dont le pendant dans la Théorie générale est «l'état de confiance ». L'obtention de la relation de probabilité ne constitue qu'une partie du processus décisionnel. En effet, nous avons vu que cette relation n'a de sens que par rapport à un capital de connaissances qui a été appelé «h». A cet égard, le lien logique entre propositions que l'agent a réussi à induire des données observables peut être plus ou moins crédible eu égard à la quantité d'informations pertinentes dont il dispose. Est-ce que « $\mathrm{h} »$ est suffisamment fourni pour accorder une quelconque crédibilité à son jugement ? Il apparaît que ce poids est de nature à infléchir une décision quel que soit le lien d'implication obtenu. Dans une situation où un fort lien d'implication logique est établi alors même que la quantité d'informations qui supporte cette relation est faible, l'agent sera enclin à n'accorder que peu de crédibilité à ce jugement.

Sur la question de la mesure du poids, Keynes n'avance que peu de choses, sicen'est que l'on ne peutétablir de comparaisons que dans un nombrelimité de cas :«This may be possible when the conclusion of the two arguments is the same, and the relevant evidence in the one includes and exceeds the evidence in the other... When the conclusion of two arguments are different will often be impossible to compare their weight » [1921, p. 80].

Enfin, il est important pour notre propos de souligner que Keynes assigne également une dimension objective au poids des arguments. La question de savoir comment le poids des arguments vient infléchir la décision ne fait pas l'objet d'une analyse précise de la part de Keynes. Si l'on pose une série d'arguments $(\mathrm{a} / \mathrm{h} 1),(\mathrm{a} / \mathrm{h} 2), \ldots,(\mathrm{a} / \mathrm{hN})$, où $\mathrm{hN}=\mathrm{hN}-1+\mathrm{i}^{*}$ avec $\mathrm{i}^{*}$ une information additionnelle pertinente, Keynes postule que l'individu rationnel fonde sa décision sur $(\mathrm{a} / \mathrm{hN})$, à savoir la relation dont le poids est le plus important.

La théorie keynésienne des anticipations étant posée, il est possible à présent d'élucider les liens entre la théorie du Treatise et les anticipations telles que posées dans la Théorie générale. 
1.2 Le traitement des anticipations dans la Théorie générale : une apparente identité de vue avec le Treatise on probability

Keynes ne présente pas, dans la Théorie générale, une reformulation de sa théorie des anticipations : la chose relèverait, eu égard au Treatise on probability consacré entièrement à la question, une pure redite. On trouve néanmoins de façon apparente la reprise des éléments fondamentaux du processus du décision analysés en 1921, dans les chapitres de la Théorie générale traitant des prévisions et du marché de la monnaie.

Les anticipations de court et long terme présentent des caractéristiques communes que nous présentons dans un premier paragraphe. Les caractères les distinguant sont exposés dans un second temps. Dans les deux cas, les anticipations de la Théorie générale semblent, en première analyse, être marquées du sceau de l'analyse du Treatise.

La base sur laquelle s'effectuent les prévisions de court comme de long terme sont les données présentes récoltées par le décideur. Ce dernier n'utilise pas l'ensemble des données dont il peut disposer, mais uniquement les seules données présentes jugées comme pertinentes. A cet égard, Keynes [1937] écrit que le présent constitue pour le futur un meilleur guide bien meilleur que ne le révèlerait un examen lucide des expériences passées. En ce qui concerne les anticipations de court terme, les résultats les plus récents jouent fréquemment un rôle prédominant dans la détermination du contenu de ces prévisions. De même, dans le champ de la longue période, on peut également lire sous la plume de Keynes [1936] que la situation présente est projetée dans le futur. On retrouve ici l'ancrage empirique des prévisions, qui se fondent sur la base d'un capital d'informations (les prémisses « $\mathrm{h} » \mathrm{du}$ Treatise), étranger à l'approche subjectiviste qui retient comme seul critère de rationalité la cohérence interne du système de prévisions.

Par ailleurs, l'agent intègre dans la formulation de son anticipation des changements dans la mesure où il a des raisons objectives, fondées, de les envisager. Dans le cadre des prévisions courtes, ce n'est que lorsque «les producteurs n'ont pas de raisons définies d'attendre un changement [qu'ils] fondent leurs prévisions sur l'hypothèse que les résultats les plus récemment réalisés se poursuivront dans l'avenir »[1936, p. 75]. En ce qui concerne les décisions liées à l'investissement, la projection dans le futur s'opère après modification «dans la seule mesure où l'on a des raisons plus ou moins précises d'attendre un changement »[1936, p.164].

Enfin, aussi bien pour les prévisions à courte portée que pour la longue période, les décisions sont contingentes au poids qu'accordent les agents à leurs propres calculs. Si des changements à venir sont très probables mais que les informations disponibles ne suffisent pas à étayer ce point de vue, les agents excluent ce calcul pour la prise de décision. Le poids des arguments permet ainsi à l'agent de choisir, parmi les calculs qu'il a pu établir, la relation de probabilité dans laquelle il a le plus confiance.

En vue d'éclaircir notre interprétation des propositions de l'auteur, résumons les termes du problème qui se posent au décideur.

Au chapitre exposant le principe de la demande effective, Keynes écrit en note de bas de page la chose suivante : 
«Un entrepreneur ayant à prendre une décision pratique relative à son échelle de production (et/ou à l'échelle de son équipement, précisé par nous) ne fait évidemment pas, au sujet du «produit » éventuel de la vente de chaque volume de production (et/ou chaque quantité de capital, précisé par nous), une prévision unique exempte d'incertitude, mais plusieurs prévisions incertaines plus ou moins probables et incertaines » [Keynes, 1936, p. 52].

Autrement dit, pour chaque volume de production pour les anticipations de court terme, et pour chaque volume de capital investi pour celles de long terme, les entrepreneurs établissent une série de prévisions plus ou moins probables. A chaque capital informationnel « $\mathrm{h} »$ (données du passé récent et présentes) est associé une série de conclusions «a » possibles et donc, une série de probabilités reliant chaque ensemble de conclusions à ce même « $\mathrm{h} »$.

Les deux grandes sources d'incertitude auxquelles doit faire face le décideur sont le degré de connaissance de la relation $(\mathrm{h} / \mathrm{a})$, ou, autrement dit, le degré d'implication qui relie les conclusions projetées « $\mathrm{a} »$ au savoir initial « $\mathrm{h} »$, et d'autre part la quantité d'information « $\mathrm{h} »$ susceptible d'étayer cette même relation. A ces deux sources d'incertitude correspondent deux types d'opérations, que sont la production de la relation de probabilité et leur pondération par le poids des arguments ou état de confiance respectivement.

Par convention, l'entrepreneur va accorder un poids plus important aux relations de probabilités ayant pour base de calcul les informations présentes. Dit formellement, l'entrepreneur privilégiera une relation de probabilité qui, pour une même prévision «a », contient un capital informationnel « $\mathrm{h} »$ plus fourni en données présentes. Il obtient alors pour chaque niveau de production ou de capital une prévision plus ou moins probable de recettes. Il sélectionne enfin son niveau de production et/ou d'investissement selon la règle du principe de la demande effective dans le premier cas, et de l'égalisation de l'efficacité marginale du capital et du taux d'intérêt dans le second.

Les caractéristiques générales des anticipations étant posées, il est à présent possible d'entrer dans les détails de ce qui sépare les anticipations de courte et de longue portée.

Le propre des prévisions de courte période est la quasi-identité entre les résultats passés les plus récents et la projection dans le futur proche. Le futur immédiat n'étant jamais que le prolongement de la situation présente, la révision de celle-ci se fait d'une façon graduelle, continue, à la lumière des résultats acquis (Keynes, [1936]).

Nous pouvons noter dans plusieurs passages de la Théorie générale que Keynesneutralise l'importance de ces prévisions en considérant qu'elles sont toujours vérifiées.

Nous avons vu que Keynes, dans le cadre du chapitre 12, ne traite que de l' "état de confiance », c'est-à-dire de l'étape ultime du schéma décisionnel qu'il a dressé. A cet égard, l'auteur de la Théorie générale ne nous renvoie, en note de bas de page, qu'au seul chapitre du Treatise consacré au «poids des croyances ». Cette référence exclusive au «weight of arguments » ne signifie pas, comme nous avons eu l'occasion de le voir dans des paragraphes précédents, que Keynes occulte dan la Théorie générale les deux premières phases d'élaboration de la relation de probabilité. Ainsi pouvons-nous dire de manière symétrique que le chapitre 11 est entièrement consacré à ce que nous avons appelé les phases 1 et 2 du processus de décision keynésien. Keynes, dans les chapitres 11 et 12, ne traite que d'une chose : l'efficacité marginale du capital. Dans un premier temps, il expose les éléments de calcul de l'entrepreneur, et par là-même, les facteurs susceptibles de faire varier sa prévision. 
Autrement dit, il mentionne les informations pertinentes dont les entrepreneurs font la collecte (1è phase) pour établir des relations logiques entre propositions et ainsi déterminer ce taux de rendement escompté qu'il nomme «efficacité marginale du capital » (phase 2). Dans le chapitre 12, Keynes introduit l'étape de la pondération des prévisions par l'état de confiance («weight of argument») des entrepreneurs, dans sa relation avec celle qui s'établit sur le marché financier.

Le calcul de l'efficacité marginale du capital recouvre trois étapes bien connues, que sont l'établissement des recettes monétaires escomptées sur la durée de vie envisagée du capital, l'actualisation de ces dernières et enfin l'égalisation de la somme des revenus actualisés au prix d'offre du capital ou coût de remplacement. Keynes mentionne les éléments pertinents que l'entrepreneur prend en compte dans son calcul, à savoir, entre autres, l'évolution du taux d'intérêt, du niveau général des prix, du prix d'offre du capital. Il ajoutera, dans ses notes sur le cycle, l'écart entre les prévisions de ventes et leur réalisation effective.

Keynes évoque ainsi les informations dont les entrepreneurs font la collecte et les variables dont ils font la prévision. Il explicite ce dont relève le calcul strict du taux de rendement escompté et se rattache, de cette manière, aux deux premières phases de la décision que l'on trouve dans le Treatise on probability.

Cependant, comme le souligne Keynes dans la Théorie générale et le TP, l'efficacité marginale du capital dépend également de la confiance dont les entrepreneurs investissent leurs propres prévisions. Ce dernier est «gonflé » par un usage selon lequel les informations présentes constituent la base la plus solide des prévisions.

Nous venons ainsi de montrer en quoi les relations entre le Treatise on probability et la Théorie générale semblent apparemment évidentes. Selon Arrous [1982], le Treatise on probability est un aspect incontournable du fondamentalisme keynésien. Pour Ventelou [1997], l'ouvrage sur les probabilités permet mieux que le Treatise on money de comprendre la Théorie générale. Selon Postel [2000], la théorie des probabilités de Keynes est au cœur de sa conception de la rationalité que l'on retrouve développée dans la Théorie générale. Selon Favereau, le traité des probabilités est une des clés de l'avenir du projet radical, dans la continuité de la Théorie générale, à travers le concept de convention.

Une partie de la littérature anglo-saxonne portant sur le sujet partage également cette vision. Ainsi, Carabelli [1988] explique dans son ouvrage la cohérence et l'unité des différents travaux de Keynes. Elle défend l'idée, comme exposé plus haut, que les chapitres 11 et 12 de la Théorie générale peuvent être relus sous un angle nouveau grâce au Treatise on probability. Lawson [1985, 1988] considère à l'instar des économistes français que les apports théoriques de Keynes dans les deux ouvrages fournissent le fondement à une théorie de la décision en situation d'incertitude. O'Donnell [1989] soutient également la thèse de la continuité entre le Treatise et la Théorie générale.

Pourtant, cette analyse est selon nous erronée : elle relève d'une interprétation faussée du Traité des probabilités. 


\section{Treatise on probabilityetThéorie générale : l'absence de lien}

Nous avons vu dans le précédent chapitre qu'un lien fort entre les ouvrages de 1921 et 1936 pouvait être établi. En substance, nous avons identifié d'une part un concept commun de convention et d'autre part une théorie de la décision présente dans le premier ouvrage venant compléter l'analyse développée dans le second. L'idée qu'il existerait un concept de convention dans le Treatise pouvant être transposé à la Théorie générale est pourtant loin d'être évidente. Une telle identification est non seulement susceptible de conduire à une impasse, mais n'a en outre pas de légitimité, au sens où la théorie de Keynes l'interdit. Plus fondamentalement, loin d'être proches, c'est un gouffre qui sépare les deux œuvres : si le Treatise a pour objet d'analyse l'incertain logiquement probabilisable, la Théorie générale traite d'incertitude radicalement non-probabilisable.

2.1 L'impossible transposition de la « convention » du Treatise à la convention supposée de la Théorie générale

L'analyse du chapitre précédent nous a conduit à reconnaître dans les prémisses informationnelles de la relation de probabilité le fondement du concept de convention exposé dans la Théorie générale. Cependant, cette assimilation ne va pas sans poser de problèmes compte-tenu de l'analyse développée dans le chapitre 12 de l'ouvrage de 1936.

Rappelons avant toute chose la façon dont Keynes introduit le concept de convention dans la Théorie générale.

A la question « comment donc s'effectuent dans la pratique ces réévaluations d'une si haute portée auxquelles les investissements existants sont soumis tous les jours et même toutes les heures » [1936, p. 163], Keynes répond que « nous sommes tous convenus, en règle générale, d'avoir recours à une méthode qui repose à vrai dire sur une véritable convention. Cette convention consiste essentiellement dans l'hypothèse que l'état actuel des affaires continuera indéfiniment » [Ibid, p. 164]. Cette hypothèse conventionnelle « est compatible avec un haut degré de continuité et de stabilité dans les affaires, tant que l'on peut compter sur le maintien de la convention » [Ibid, p. 163]. D'autre part, " nous supposons, en vertu d'une véritable convention, que l'évaluation actuelle du marché est la seule correcte, et que la dite convention variera seulement dans la mesure où cette connaissance sera modifiée » [Keynes, 1936, p. 168-169].

Le texte de Keynes mentionné ci-dessus fait apparaître trois idées à propos de la convention financière :

- il s'agit d'une " méthode de calcul », basée sur l'hypothèse que " l'état des affaires continuera indéfiniment " ; c'est une grille d'analyse, un instrument de formation des anticipations commun aux agents ;

- elle permet de lever l'instabilité inhérente au fonctionnement du marché financier ; la référence « extérieure » que constitue la méthode, permet aux agents de sortir du mécanisme auto-référentiel ;

- elle est assortie d'une croyance commune, appelée également par Keynes «convention» selon laquelle l'évaluation du marché est correcte, même si chacun sait qu'elle ne l'est pas nécessairement. 
Orléan la définit, en s'appuyant sur les écrits de Keynes, comme « une organisation sociale au travers de laquelle la communauté se dote d'une référence commune, produit d'une représentation commune qui fonde les anticipations individuelles » [1990, p. 265].

Supposons donc que la communauté des acteurs du marché financier dispose d'un capital de prémisses commun « $\mathrm{h}$ » et qu'ils estiment sa relation d'implication d'avec « a » le vecteur des prix futurs des actifs du marché financier.

Puisque les agents anticipent tous la même chose ( a $»)$, compte-tenu des mêmes prémisses « $\mathrm{h}$ », puisque conventionnelles, les relations de probabilité ne peuvent qu'être les mêmes. Pour Keynes en effet, même si la relation de probabilité n'est pas donnée à l'agent économique qui la formule, elle n'en demeure pas moins objective et donc unique : «A proposition is not probable becausewethinkso. When once the facts are given which determine our knowledge, what is probable or impropable in these circumstances has been fixed objectively, and is independent of our opinion» [1921, p.18].

Lawson [1988] considère à cetégard que la conception keynésienne des probabilitésdéveloppéedans le Treatise et la Théoriegénérales'opposefrontalement à l'optique des probabilitéssubjectives de Ramsey: «The difference between Keynes's theory and the subjective view, clearly, is that although both accept that probability exists only on the level on knowledge or opinion, Keynes believes that probability is objectively determined » (p.44).

L'unicité des prémisses et des conclusions implique celle de la probabilité; il s'agit là d'un enseignement majeur du Treatise. Dans une telle configuration, les agents vont donc tous anticiper la même chose. Tous voudront acheter et vendre les mêmes titres. La convention manifeste alors un état de non-coordination totale. La signification de cette absence de compatibilité entre les plans de décisions individuels est l'illiquidité du marché, c'est-à-dire précisément ce que la convention est censée lever. Il s'avère donc que les prémisses informationnelles ne sauraient être transposées à une analyse en termes de coordination économique par la convention.

Restent néanmoins deux éléments de la théorie keynésienne des anticipations qui pourraient donner du poids au point de vue conventionnaliste.

Tout d'abord, la probabilité d'une même proposition logique peut varier (selon Keynes) selon les individus, soit en raison d'une différence dans le capital cognitif des agents, soit à cause de capacités mentales d'analyse inégales. C'est la seconde cause qui sera retenue puisque le corps de connaissances est supposé, pour l'instant, parfaitement homogène puisque conventionnel. Ensuite, la notion de «poids des arguments » vient pondérer la probabilité dans un sens ou un autre la décision.

Nous pouvons d'ores et déjà balayer la seconde proposition, puisque le modèle d'Orléan [1992] établit un étroit entre l'état de confiance et le degré de consensus sur les probabilités établies. Autrement dit, lorsque les vues sur l'avenir s'accordent parfaitement, leur confiance en la convention tend à être totale et donc homogène (même s'il existe quelques agents marginaux appelés « contrarians »).

Pour Orléan en effet, la transition d'une anticipation à une autre dépend de la fréquence d'opinion constatée sur le marché financier et de la confiance relative des agents en leur propre estimation. L'économiste considère que la confiance accordée à l'opinion des autres 
dépend de la fréquence observée de cette opinion : plus elle est élevée, plus la confiance accordée à l'opinion extérieure l'est aussi [Orléan, 1992]. Il faudrait donc lever la relation fonctionnelle et bi-univoque entre état de l'opinion et confiance, ce qui ruinerait la mécanique du modèle.

Le premier argument évoqué pour rétablir la cohérence de l'interprétation conventionnaliste appelle l'analyse des éléments subjectifs entrant dans la formation des décisions chez Keynes.

Il peut être décelé deux éléments subjectifs dans l'établissement de la relation de probabilité chez Keynes. A la page 4 du Treatise, ce dernier dit que «whatparticular propositions select on the premisses of our argument naturallydepend on subjective factorsparticular to ourselves ». Le stock d'informations recueilli par les agents dépend de considérations d'ordre personnel. Tout le monde ne dispose pas forcément du même stock d'informations pour effectuer le même type de décision. Une variante dans les prémisses, qu'elle soit purement quantitative, purement qualitative ou encore les deux à la fois, suffit à modifier la relation de probabilité objective. Il s'agit là d'un facteur subjectif agissant en amont de la prise de décision, puisque son champ d'action est celui de la récolte des connaissances directes.

Le second élément par contre est de nature à pondérer, a priori, notre point de vue. Pour Keynes en effet, si la relation de probabilité est unique, il n'en reste pas moins qu'elle n'est pas une donnée pour l'agent, et par voie de conséquence, le décideur doit la découvrir. Or, la capacité intellectuelle d'analyse et d'induction des agents n'est pas homogène. La probabilité sur la base de laquelle un agent prend une décision peut différer de celle d'un autre agent même si les deux prévisions sont objectivement identiques. Néanmoins, Keynes relativise cette hétérogénéité en distinguant schématiquement deux groupes d'individus, du point de vue de leur possibilité d'analyse et de réflexion.

Il y aurait d'un côté ceux qui, éclairés, obtiennent la relation objective, et de l'autre, ceux dont les capacités sont restreintes, qui feront des erreurs de jugement. Autrement dit, une capacité intellectuelle minimale est requise pour atteindre la probabilité objective. En-deçà de cette capacité, une même probabilité objective sera obtenue avec une marge d'erreur qui dépend des capacités intellectuelles des individus. Il est important donc de noter qu'une et une seule relation de probabilité sera découverte :

- sans erreur si la capacité minimale requise est atteinte ;

- avec erreur si tel n'est pas le cas ; mais une différence entre deux individus ne peut résulter que de différentes capacités mentales d'induction. Deux individus dotés de la même capacité ne peuvent logiquement que trouver une relation identique.

Tel est le cas parce que la relation de probabilité ne dépend pas des préférences individuelles des agents, de leur opinion personnelle, ou encore de leur humeur du moment. La relation de probabilité compte-tenu d'un capital cognitif donné, ne peut être qu'unique. Ainsi, à une même capacité mentale correspondra une même « découverte » de la relation de probabilité.

Keynes témoigne, dans le cadre de cette schématisation de la communauté des individus, d'une vision élitiste de la société. On retrouve cette séparation, présente dans le Treatise on Probability, notamment dans le chapitre 12 de la Théorie générale. Il y a deux sortes d'agents économiques du point de vue de leur capacité mentale. Tout d'abord, citons «les professionnels compétents, doués d'un jugement plus sûr et de connaissances plus étendues que la moyenne des investisseurs privés », qui se distinguent par «leur habilité ». On trouve ensuite, à l'opposé, la «masse d'un grand nombre d'individus ignorants » [1936, p. 169]. 
Cependant la communauté des acteurs sur un marché considérée par Keynes est relativement homogène du point de vue de leur capacité intellectuelle. Sur le marché financier en particulier, l'évaluation conventionnelle est gouvernée par la masse des individus ignorants. Keynes les place sur un même niveau. Par conséquent, il n'y a pas à penser que la relation de probabilité obtenue par des individus tous aussi ignorants les uns que les autres, varierait alors même que leur capital cognitif est identique et que l'objet de l'anticipation est commun à tous.

De même, l'entrepreneur qui réalise son « activité d'entreprise », en établissant le barème de l'efficacité marginale du capital, fait partie de cette communauté homogène des acteurs « éclairés ». Ces derniers, bien qu' « ayant des aptitudes et des caractères différents » sont supposés avoir cette capacité minimale requise: ces derniers sont doués d' "esprits animaux », ayant le goût du risque mais aussi l'intuition du fonctionnement de l'économie.

Ainsi, l'auteur sépare deux catégories d'individus et a tendance à considérer ces communautés comme homogènes. Or, le seul élément pouvant faire varier une probabilité objective d'un individu à un autre, étant donné «a » et «h», est la capacité mentale individuelle. Les considérations de Keynes interdisent donc de penser qu'il y ait un quelconque concept de convention dans l'analyse du Treatise on probability.

2.2 Treatise on probability et Théorie générale : deux cadres d'analyse mutuellement exclusifs

Le précédent développement contribue à mettre en doute le lien analytique entre les deux ouvrages de Keynes. Certains économistes postkeynésiens rejoignent notre analyse. Selon eux en effet, il existe une rupture entre la Treatise et la Théorie générale, contre l'avis de Lawson [1988], Carabelli [1988], O’Donnell [1989], ou encore Roncaglia [2009].

Ainsi, pour Bateman [1987, 1996] et Davis [1994], Keynes abandonne sa théorie développée dans le Treatise à la lumière des travaux de Ramsey, qui développe une conception subjective des probabilités. Pour Bateman [1987], «while he (i.e. Keynes ) had originally advocated an objective epistemic theory of probability in A Treatise on Probability, he was now willing to accept a subjective epistemic theory» (p. 107). A cetégard, Gillies [2003] précise la chose suivante:

« As far as the interpretation of probability is concerned, a most important intellectual event took place between 1921 and 1936. As we have seen in the previous section, Ramsey in his 1926 paper 'Truth and Probability' subjected Keynes's logical interpretation of probability to an extensive criticism. There is strong evidence that Keynes, who had the greatest respect for Ramsey, took this criticism very seriously, and altered his views on probability in the light of Ramsey's objections » (p.10).

Keynes se serait donc détourné d'une vision objective des probabilités (développée dans le Treatise) au profit d'une vision subjective (développée dans la Théorie générale).

La thèse que nous défendons partage cette idée de rupture entre les deux ouvrages mais pour des raisons différentes: indépendamment de toute modification dans la conception keynésienne des probabilités, c'est la différence de cadres d'analyse qui explique l'absence de lien entre le Treatise et la Théorie générale. 
Pour le montrer, il est nécessaire de rappeler ce à quoi Keynes s'oppose dans le Treatise on probability.L'économiste remet en cause les deux grandes traditions en matière de théorie des probabilités : l'approche mathématique et l'approche fréquentiste.

La première (l'approche mathématique) repose sur ce que Keynes appelle le principe d'indifférence, qui n'est jamais que le principe de raison non-suffisante de Bernouilli. La probabilité est définie, selon ce dernier, comme le rapport entre le nombre d'alternatives favorables et le nombre total d'alternatives. Or dit Keynes, une telle loi ne peut être valide en général. Elle requiert que les probabilités puissent être mesurées ou comparées. Si tel n'est pas le cas, le principe d'indifférence n'a plus lieu d'être. Parmi les grands types de relations de comparaison possibles entre les probabilités (comparaisons cardinale, ordinale, et noncomparabilité), le troisième est le plus fréquent, en raison de l'hétérogénéité des ensembles d'informations récoltés par l'individu. Ces types de comparaisons ont pour origine l'existence de deux sous-classes de probabilités :

- les probabilités numériques, inséparables d'un espace fini d'alternatives équiprobables, exclusives et indivisibles ;

- les probabilités non-numériques, ensemble de relations non-comparables en raison de l'absence d'unité de mesure commune.

Keynes, dans le chapitre 3 du Treatise, explique que le caractère qualitatif des ensembles d'information n'est pas réductible à leur caractère quantitatif. Il prend trois exemples pour illustrer son propos, celui des couleurs, des nombres cardinaux et de l'analogie :

\footnotetext{
«When we describe the colour of one object as bluer than that of another, or say that it has more green in it, we do not mean that there are quantities blue and green of which the object's colour possesses more or less ; we mean that the colour has a certain position in an order of colours and that it is nearer some standard colour than is the colour with which we compare it » [1921, p. 38].
}

«We say that the number three is greater than the number two, but we do not mean that these numbers are quantities one of which possesses a greater magnitude than the other. The one is greater than the other by reason of its position in the order of numbers » [Ibid, page 39].

«When we say of three objects A, B and C that B is more like A than C, we mean, not that there is any respect in which $B$ is in itself quantitatively greater than $C$, but that B is nearer to $A$ than $C$ is. There are also, as in the case of probabilities, different orders of similarity » [Ibid, p. 39].

Ainsi, il est des propositions dont la mise en rapport arithmétique est impossible. En outre, la comparaison entre les probabilités les caractérisant n'a aucun sens, car elles n'appartiennent pas à un espace commun de mesure : «Probabilities do not belong to a single set of magnitudes measurable in terms of a common unit » [Ibid, p. 33].

L'approche fréquentiste des probabilités repose quant à elle sur la mise en évidence de lois empiriques. La probabilité d'un évènement futur est établie sur la base des lois statistiques qui ont gouverné les évènements passés. L'incertitude est réduite ici à un simple défaut de connaissance empirique. Or selon Keynes, si l'étude du passé est à même d'augmenter notre stock de connaissances, en aucun cas elle n'autorise la perception des évènements futurs dans la mesure où le monde social n'évolue pas dans l'uniformité et la répétition infinie des mêmes faits. Le propre de l'action humain, qui est de faire surgir le nouveau, d'une part interdit de considérer la répétitivité des phénomènes en vertu de lois statistiques, et d'autre part empêche le pré-établissement d'une liste d'états de la nature auxquels sont attribués des probabilités. Keynes perçoit la probabilité comme une procédure de raisonnement sur la base de propositions, et non comme une simple mise en relation d'évènements [Ibid, p. 35]. 
L'objet du Treatise n'est finalement pas tant de rejeter les approches traditionnelles que de les englober dans une théorie qui se veut plus générale. A cet égard, Keynes souhaite étendre le champ de la théorie des probabilités aux domaines de la vie sociale pour lesquels, comme il vient d'être montré, les approches fréquentistes et mathématiques sont généralement inopérantes. Il ne s'agit donc pas d'affirmer, dans le Treatise, que les évènements ne sont pas, en général, probabilisables. Il s'agit au contraire de construire une théorie des probabilités apte à s'appliquer dans un nombre de configurations plus important que ne l'autorisent les approches habituelles. La spécificité de cette théorie est

-de ne pas faire opposition entre hasard et déterminisme, mais entre hasard et connaissance, -de faire de la probabilité une relation qui peut être non-numérique, mais, dans tous les cas, exprimant une relation causale affaiblie, et représentant ainsi un opérateur de connaissance.

Il ne faut donc pas faire l'amalgame entre le non-mesurable, souligné par Keynes dans le Treatise, et le non-probabilisable. Dans le cadre d'analyse du Treatise, l'incertitude ne fonde pas le non-probabilisable mais le non-mesurable. Or, comme nous le verrons, Keynes retient dans la Théorie générale une catégorie d'incertitude qui engendre le non-probabilisable.

Dans le deuxième chapitre du Treatise on probability («probability in relation to the theory of knowledge »), Keynes établit deux types de classifications de la connaissance. Il distingue, comme nous l'avons vu précédemment, la connaissance directe de la connaissance indirecte, mais aussi les degrés de connaissance. Sont ainsi recensées la connaissance parfaite ( «properknowledge »), la connaissance incomplète ( incompletedknowldge »), et la connaissance vague ( «vague knowledge »). La connaissance parfaite relève d'une perception sans faille des liens logiques unissant les ensembles de propositions [1921, p. 14]. La connaissance incomplète résulte d'insuffisances soit dans les capacités mentales de l'individu décideur, soit dans l'ensemble d'informations dont il peut disposer. L'objet du Treatise on probability est précisément de fonder une théorie logique des probabilités dans les cas de connaissance parfaite et incomplète. En revanche, la connaissance vague, à partir de laquelle il est impossible d'inférer une quelconque relation logique, est exclue du cadre d'analyse du Treatise. Keynes affirme en effet que rien ne peut être dit dans ce cas spécial d'incertitude, ou du moins, qu'il n'est pas capable d'en faire théorie :

«I cannot attempt here to analyse the meaning of vague knowledge. It is certainly not the same thing as knowledge proper, whether certain or probable, and it does not seem likely that it is susceptible of strict logical treatment. At any rate I do not know to deal with, and in spite of its importance, I will not complicate a difficult subject by endeavouring to treat adequately the theory of vague knowledge» [1921, pp. 17-18].

Illustrons à l'aide d'un exemplaire tiré de Georgescu-Roegen [1958] ces différentes situations d'incertitude. Soit l'énoncé suivant :

E1. Une urne $U$ contient $1 / 3$ de boules rouges et $2 / 3$ de boules noires.

E2. Une urne U, sur les 3426000 tirages effectués avec remise dans l'urne, permet d'obtenir $1 / 3$ de boules rouges et $2 / 3$ de boules noires.

E3. Une urne U, sur les 10 tirages effectués avec remise dans l'urne, permet d'obtenir $1 / 3$ de boules rouges et $2 / 3$ de boules noires.

E4. Une urne U contient des boules.

L'individu qui tire une boule de l'urne souhaite établir sa chance de tirer une boule de couleur déterminée. Avec les énoncés E1 et E2, l'individu est dans un cas de «properknowledge ». Il peut représenter cet aléa par les probabilités 1/3-2/3. L'énoncé E3 
plonge l'individu dans une situation d'incertitude ou d' " incompletedknowledge ». L'acteur ne peut pas rigoureusement fonder une relation de probabilité à partir des données de l'expérience. Le jugement de probabilité est possible, mais ne peut être assis sur une approche empirique ou mathématique. L'individu doit, compte-tenu des informations dont il dispose, établir un « degré de croyance rationnel », ou, autrement dit, un degré d'implication logique, sur la base de son propre raisonnement. Par contre, l'énoncé E4 place le décideur dans une configuration d'incertitude radicale ou encore de "vague knowledge », dans le cadre de laquelle rien ne peut être dit. Le processus de décision, dont il apparaît difficile de faire la théorie, est totalement arbitraire.

Ceci étant posé, voyons à présent quel le type d'incertitude est à l'œuvre dans la Théorie générale.

Keynes multiplie les allusions, dans le chapitre 12, à une incertitude dont l'épaisseur est impénétrable. La prévision de long terme ne semble pouvoir reposer sur aucune donnée tangible. Keynes souligne que la quantité d'informations sur lesquelles les agents peuvent logiquement s'appuyer pour former leur prévision, ou, autrement dit, leurs relations de probabilité, est si infime que l'anticipation relève de la pure prophétie.

« Le fait marquant en la matière est l'extrême précarité des bases sur lesquelles nous sommes obligés de former nos évaluations des rendements escomptés. Notre connaissance des facteurs qui gouverneront le rendement d'un investissement quelques années plus tard est en général très frêle et souvent négligeable. A parler franc, on doit avouer que, pour estimer dix ans ou même cinq ans à l'avance le rendement d'un chemin de fer, d'une mine de cuivre, d'une fabrique de textile, d'une marque pharmaceutique, d'un transatlantique ou d'un immeuble dans la City à Londres, les données dont on dispose se réduisent à peu de choses, parfois à rien » [1936, p.165].

Le propos de Keynes est ici de nier toute possibilité de calculer le rendement futur du capital et de probabiliser l'avenir : «le calcul exact des bénéfices à venir joue un rôle à peine plus grand que dans une expédition au Pôle Sud» [1936, p. 176]. L'auteur n'affirme donc pas simplement l'impossibilité, compte-tenu de l'incertitude et de la nature des évènements futurs à mettre en relation, de l'impossible mesure et comparaison des probabilités. Il affirme avec vigueur l'impossibilité d'établir des relations de probabilités. Keynes l'exprime clairement dans l'article du QJE de 1937 : «Par connaissance incertaine, je m'explique, je n'entends pas simplement distinguer ce que l'on sait pour certain de ce qui est probable [...]. Nous ne savons tout simplement pas » [p 115].

Sans l'ombre d'un doute, Keynes traite dans la Théorie générale du cas de «vague knowledge » auquel il consacre à peine trois lignes dans le Treatise on probability. Ce dernier ouvrage traite précisément d'une situation de «properknowledge». Autrement dit, l'incertitude dont Keynes traite dans l'ouvrage de 1936 est insondable, et n'a rien à voir avec le cadre d'analyse du livre de 1921 dont l'objet, comme son titre l'indique, est la construction d'une théorie de probabilités. Que Keynes ait pu retenir le concept de poids des arguments pour distinguer le probable de l'incertain est une chose. Qu'il faille voir dans la Théorie générale un lien fort de continuité avec le Treatise on Probability dont l'unité serait l'incertitude en est une autre, et demeure inacceptable.

\section{Conclusion}

Alors même que les liens entre Traité des probabilités et Théorie générale semblent évidents, il nous semble important de revisiter les deux ouvrages pour comprendre que leurs objets d'étude respectifs sont différents. Il n'y a pas à proprement parler d'unité de la révolution 
keynésienne reposant sur sa théorie des anticipations et de l'incertitude. Keynes développe en effet en 1921 une théorie de la connaissance et de la décision qui possède un cadre totalement différent de celui considéré dans la Théorie Générale et les articles qui lui sont postérieurs. C'est peut-être pour cela qu'un tel projet de développement de l'approche fondamentaliste n'a pas connu le succès annoncé par certains.

\section{Bibliographie}

Arrous Jean, [1982], «Keynes et les probabilités: un aspect du fondamentalisme keynésien », Revue Economique, n5, p. 839-861.

Barrère Alain., [1990], Macroéconomie keynésienne, Dunod, Paris.

Bateman Bradley [1987], « Keynes's Changing Conception of Probability », Economics and Philosophy, 3, p. 97-120.

Bateman Bradley, [1996], Keynes's Uncertain Revolution, University of Michigan Press.

Carabelli Anne, [1988], On Keynes's Method, MacMillan, London.

Davis John Brian, [1994], Keynes's Philosophical Development, Cambridge University Press.

O’Donnell Rodd, [1989], Keynes : philosophy, Economics and Politics, MacMillan, London.

Gillies Donald, [2003], « Probability and uncertainty in Keynes's General theory », working paper.

Faverau Olivier, [1985], «L'incertain dans la révolution keynésienne », Economies et Sociétés, Série PE n³, mars, p. 29-72.

Favereau Olivier, [1988], « De l'économie conventionnelle à l'économie des conventions », Cahiers d'Economie politique, janvier, n¹4-15, p. 197-220.

Georgescu-Roegen Nicholas, [1958], « The nature of expectation and Uncertainty », in Georgescu-Roegen Nicholas, [1966], Analytical Economics, Harvard University Press.

Keynes John Maynard, [1921], Treatise On Probability, tome VIII des Collected Writtings of John Maynard Keynes, [1971], Royal Economic Society, MacMillan-CUP, London.

Keynes John Maynard, [1930], Treatise On Money, tomes V et VI des Collected Writtings of John Maynard Keynes [1971], Royal Economic Society, MacMillan-CUP, London.

Keynes John Maynard, [1936], Théorie générale de l'emploi, de l'intérêt et de la monnaie, Payot, Paris.

Keynes John Maynard, [1937], «La théorie générale de l'emploi », QuarterlyJounral Of Economics, vol. 51, republié dans The CollectedWrittings [1971], vol XIV, p.109-123. 
Le Heron Edwin, Asensio Angel, Charles Sebastien, Dany Lang, [2011], «Les développements récents de la macroéconomie post-keynésienne », Revue de la régulation, $\mathrm{n}^{\circ} 10$, second semestre.

Lawson Tony, [1988], «Probability and uncertainty in Economic Analysis », Journal of Post-Keynesian Economics, vol 11, n¹, p. 38-65.

Lawson, Tony, [1985], «Uncertainty and Economic Analysis », The Economic Journal, 95, p. 909-27.

Lewis David, [1969], Convention, A philosophical study, Cambridge Mass, Harvard University Press.

Orio Lucien, Quiles Jean José, [1994], L'économiekeynésienne : un projet radical, Editions Nathan, collection Circa, Economie Sciences sociales.

Orléan André, [1999], Le pouvoir de la finance, éditions Odile Jacob, Paris.

Orléan André, [1994], Analyse économique des conventions, PUF, Economie, Paris.

Orléan André, [1992], «Contagion des opinions et fonctionnement des marchés », Revue Economique, vol.43, n4, p. 685-697.

Orléan André, [1990], « Le rôle des influences interpersonnelles dans détermination des cours boursiers », Revue Economique, vol. 41, n5, p. 839-868.

Orléan André, [1988], «L'auto-référence dans la théorie de la spéculation », Cahiers d'Economie Politique, $\mathrm{n}^{\circ} 14-15$, p. 229-242.

Ramsey, Franck Plupton, [1926], Truth and Probability, in Ramsey, [1931], Foundations of Mathematics and other Logical Essays, R.B. Braithwaite, London, p.156-98.

Roncaglia Alessandro, [2009], «Keynes and probability: An assessment », European Journal for the history of economic thought, September, vol 16, p. 489-510.

Postel Nicolas, [2000], «Rationalité relative, jugement pratique et coordination chez Keynes », Cahiers lillois d'économie et de sociologie, p. 117-145.

Ventelou Bruno, [1997], Lire Keynes et le comprendre, Vuibert Economie, Paris. 
